INPLASY

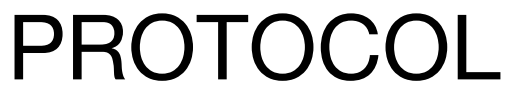

To cite: Yang et al. The effectiveness and safety of curcumin as a complementary therapy in inflammatory bowel disease: a protocol of systematic review and metaanalysis. Inplasy protocol 202090065. doi: 10.37766/inplasy2020.9.0065

Received: 15 September 2020

Published: 15 September 2020

Corresponding author:

Zhenhuan Yang

18810255933@163.com

Author Affiliation:

Beijing University of Chinese Medicine

Support: None.

Review Stage at time of this submission: Preliminary searches.

Conflicts of interest: None.

\section{The effectiveness and safety of curcumin as a complementary therapy in inflammatory bowel disease: a protocol of systematic review and meta-analysis}

Yang, Z1; Liu, W2; Zhou, X3; Zhu, X4; Suo, F5.

Review question / Objective: The objective of this study was to systematically review the literature to verify the efficacy and safety of curcumin as a complementary therapy for the maintenance or induction of remission in patients with inflammatory bowel disease (IBD).

Condition being studied: Curcumin is the main pharmacologically active curcuminoid pigment in turmeric. It acts by modulating various cell-signaling pathways, producing anti-inflammatory, anti-tumor, anti-oxidant, and immunomodulatory effects. This systematic review aims to analyze the studies published so far, to review the positive or negative effects of the use of curcumin, and to determine whether it is safe and effective as a complementary therapy in the management of IBD.

INPLASY registration number: This protocol was registered with the International Platform of Registered Systematic Review and Meta-Analysis Protocols (INPLASY) on 15 September 2020 and was last updated on 15 September 2020 (registration number INPLASY202090065).

\section{INTRODUCTION}

Review question / Objective: The objective of this study was to systematically review the literature to verify the efficacy and safety of curcumin as a complementary therapy for the maintenance or induction of remission in patients with inflammatory bowel disease (IBD).
Rationale: Inflammatory bowel disease (IBD) is a chronic condition that affects the relapsing gastrointestinal tract, with periods of exacerbation and remission. Its main forms of presentation are ulcerative colitis (UC) and Crohn's disease (CD). Its etiopathogenesis is believed to be due to a loss of tolerance to the intestinal 
microbiota associated with marked immune responses and environmental factors in genetically susceptible individuals.Currently, corticosteroids, sulfasalazine, mesalamine (5-ASA), and immunomodulators are treatment options for patients with IBD. However, it is worth mentioning that conventional treatments cause numerous side effects due to a marked immune response suppression, which negatively impacts the quality of life of these individuals. Studies indicate that a substantial proportion of patients do not fully respond to the conventional treatments for IBD, or that its efficacy wanes over time. Curcuma longa is a plant from the Zingiberaceae family that is native to India and Southeast Asia and is well known in Asian cultures. Known commonly as turmeric, it has long been used in Ayurvedic medicine to treat inflammatory diseases.

Condition being studied: Curcumin is the main pharmacologically active curcuminoid pigment in turmeric. It acts by modulating various cell-signaling pathways, producing anti-inflammatory, anti-tumor, anti-oxidant, and immunomodulatory effects.This systematic review aims to analyze the studies published so far, to review the positive or negative effects of the use of curcumin, and to determine whether it is safe and effective as a complementary therapy in the management of IBD.

\section{METHODS}

Search strategy: ( ( " Colitis, Ulcerative"[Mesh]) OR ((((Idiopathic Proctocolitis[Title/Abstract]) OR (Ulcerative Colitis[Title/Abstract])) OR (Colitis Gravis[Title/Abstract])) OR (Inflammatory Bowel Disease[Title/Abstract])) OR (Ulcerative Colitis Type[Title/Abstract]))) AND ((("Curcumin"[Mesh]) OR (((Turmeric Yellow[Title/Abstract]) OR (Yellow, Turmeric [Title/Abstract])) OR (Diferuloylmethane[Title/Abstract]))) AND (randomized controlled trial[Publication Type] OR randomized[Title/Abstract] OR placebo[Title/Abstract]).
Participant or population: Study participants in different age ranges with IBD can be included in the study without restricting nationality, sex, race, occupation, or education.

Intervention: The control group: basic treatment (including conventional medication and health education) and Placebo. The experimental group: basic treatment and curcumin.

\section{Comparator: Placebo.}

Study designs to be included: Randomized controlled trials (RCTs).

Eligibility criteria: The criteria for the inclusion of the RCTs in this study were that they used curcumin for the maintenance or remission of IBD in patients of both sexes and of any age who were in remission or who had mild or moderate activity at the time of recruitment, and that they evaluated the effects of curcumin on the inflammatory activity. Studies published in any language were accepted, and no minimum follow-up period was established. Review articles, animal studies, editorial letters, in-vitro studies, observational, and descriptive studies, such as case reports and case series, were excluded. In addition, studies that did not describe the curcumin dose or did not meet the minimum bias risk assessmentscore were also excluded.

Information sources: PubMed, Web of Science, MEDLINE, Cochrane Central Register of Controlled Trials, Springer, EMBASE, the China National Knowledge Infrastructure Database, Wan Fang Database, the Chinese Scientific Journal Database, and Chinese Biomedical Literature Database.

Main outcome(s): Clinical and remission will be evaluated as the primary outcome.

Additional outcome(s): The secondary outcomes of this review will include: 1 . Clinical response. 2. Endoscopic remission. 3. Inflammatory markers (hs-CRP, ESR, 
inflammatory factor levels) 4. Adverse events.

Data management: We will make a standard data collection sheet before data extraction. Two reviewers will independently extract data from the selected studies and fill in the data collection sheet. Discrepancies and uncertainties will be resolved by consensus between the 2 review authors or by asking the third author to make a final decision. We will extract the following data: 1. General information: the first author, title, the journal, publication type, publication year, country, fund source. 2. Methods: study design, sample size, randomization, allocation concealment, blinding methods, inclusion criteria, and exclusion criteria. 3. Participants: age, gender, severity of IBD, baseline disease activity. 4 . Interventions: type of control, duration of treatment, frequency of treatment. 5. Outcomes: primary and secondary outcomes, treatment costs, adverse effects, and follow up.

Quality assessment / Risk of bias analysis: Two review authors will independently use the criteria outlined in the Cochrane Handbook for Systematic Reviews of Interventions to assess the risk of bias in the included studies. The following 6 domains in the Cochrane "Risk of bias tool" will be assessed: random sequence generation, allocation concealment, blinding, incomplete outcome data, selective reporting, and other bias. We will grade each potential trial of bias as high, low, and unclear. Any disagreement will be resolved by discussing or by asking the 3rd author to make a final decision.

Strategy of data synthesis: RevManV.5.3.5 will be used for data analysis and synthesis. Continuous data will be expressed as MD/SMD with $95 \%$ Cls, while the dichotomous outcomes will be presented as RR with $95 \%$ Cls. When $\left.\right|^{2}<50 \%$, the fixed effect model will be adopted to analyze. Otherwise, the random effect model will be selected. Additionally, we will use the sensitivity analysis and subgroup analysis to explore the causes of heterogeneity.

Subgroup analysis: Subgroup analysis will be carried out based on the age of patients, different types of curcumin therapies, duration of treatment, frequency of treatment and the degree of IBD severity or different types of IBD.

Sensibility analysis: Sensitivity analysis will be conducted to assess the reliability and robustness of the aggregation results via eliminating trials with high bias risk. A summary table will report the results of the sensitivity analyses.

Language: English.

Country(ies) involved: China.

Other relevant information: None.

Keywords: curcumin.

Contributions of each author:

Author 1 - Zhenhuan Yang - Literature serch and screening.

Author 2 - Wenjing Liu - Literature serch and screening.

Author 3 - Xuefeng Zhou - Literature serch and screening.

Author 4 - Xianran Zhu - Data collection and analysis.

Author 5 - Feiya Suo - Data collection and analysis. 